\title{
Risk Factors for Spontaneous Intestinal Perforation in Extremely Low Birth Weight Infants ${ }^{\S}$
}

\author{
Irfan Ahmad ${ }^{1}$, Kristy F. Davis ${ }^{2}$, Sherif Emil ${ }^{*}, 2$, Cherry $\mathrm{Uy}^{1}$ and Jack Sills ${ }^{1}$ \\ ${ }^{I}$ Division of Neonatology, Department of Pediatrics, and ${ }^{2}$ Division of Pediatric Surgery, Department of Surgery Univer- \\ sity of California Irvine School of Medicine and Children's Hospital, USA
}

\begin{abstract}
Objective: Spontaneous intestinal perforations (SIP) in extremely low birth weight infants are distinctly different from necrotizing enterocolitis. The etiology of SIP is not well understood. Our objective was to identify perinatal therapeutic interventions that may increase the risk of spontaneous intestinal perforations.

Methods: Medical records of extremely low birth weight infants $(\mathrm{BW}<1000 \mathrm{~g})$ admitted to a neonatal intensive care unit during 42-month period were studied. Infants with radiologic or histologic diagnosis of necrotizing enterocolitis were excluded. Information collected included maternal and infant demographics, perinatal risk factors, clinical findings and interventions, and exposure to medications before and after delivery. Chi square and paired t-tests were used to compare SIP patients to those with no perforation (NP). Mean values are given with standard error of the mean.

Results: There were 13 SIP and 165 NP. There were more male infants $(84.6 \%$ vs $49.1 \%, \mathrm{p}<0.025)$ and more out born infants $(61.5 \%$ vs $39.9 \%, \mathrm{p}<0.05)$ in the SIP group. The use of maternal tertbutaline was higher in the SIP group (30.8\% vs $9.1 \%, \mathrm{p}<0.015)$. Early treatment with indomethacin (0-3days) was significantly higher in the SIP group $(69.2 \% v s 27.9 \%$, $\mathrm{p}=0.002)$. Hypotension requiring dopamine was significantly higher in the SIP group $(69.2 \%$ vs $34.6 \%$, $\mathrm{p}=0.017)$. Combined exposure to antenatal steroids and postnatal indomethacin was significantly higher in the SIP group (69.2\% vs $36.4 \%, \mathrm{p}=0.019)$, as was the combined early treatment with hydrocortisone and indomethacin $(7.7 \% v s 0.6 \%, \mathrm{p}=0.02)$.

Conclusions: Early use of indomethacin, and co-exposure to antenatal or postnatal steroids is related to development of spontaneous intestinal perforation in extremely low birth weight infants. Prenatal exposure to maternal terbutaline and postnatal use of dopamine for hypotension increases the risk for SIP in these infants.
\end{abstract}

Keywords: Prematurity, intestinal perforation, risk, indomethacin.

\section{INTRODUCTION}

Spontaneous intestinal perforation (SIP) is now recognized as a distinct condition that differs clinically and histologically from necrotizing enterocolitis (NEC) [1-6]. Its incidence is up to $7 \%$ in the ELBW infant population who are at highest risk $[4,5]$. The perforation typically occurs suddenly on the anti-mesenteric surface of the distal ileum [7], usually without a defined prodrome during the first two weeks of life.

The exact etiology of SIP remains unclear since it may represent the final common pathway of a group of heterogeneous conditions leading to perforation. Deficiency of enteric musculature was first suggested in 1930 [8]. Other conditions which lead to local ischemia have since been suggested and include birth asphyxia (secondary to diving reflex), umbilical catheter placement, maternal drug abuse, and twin-twin transfusion [5]. More recently, pharmacological agents have been linked to SIP, including indomethacin by itself or in combination with exogenous glucocorticoids as suggested by two randomized controlled trials of postnatal

*Address correspondence to this author at the Division of Pediatric Surgery, University of California, Irvine Medical Center, 101 The City Dr., Bldg 55, Room 110, Rte 81, Orange, CA 92868-3298, USA; Tel: (714) 4568581 ; Fax: (714) 456 8931; E-mail: semil@uci.edu

${ }^{\S}$ This paper was presented in part at the 2007 Western Society for Pediatric Research meeting in Carmel, California. glucocorticoids in preterm infants $[9,10]$. A recent multi center retrospective study [3] concurred with these findings. However, other studies including Trial of Indomethacin Prophylaxis in Preterms did not find an association between indomethacin administration and SIP [11].

Recently, it has been suggested that a patent ductus arteriosus (PDA) itself may be associated with SIP [3, 12]. Nonsteroidal anti inflammatory drugs, like indomethacin, administered to the mother have also been associated with SIP [13]. While the combined effect of exposure to antenatal steroids (AS) and postnatal indomethacin has been suggested to increase the risk for SIP, studies have remained inconclusive [14].

We report a single institution experience with SIP and perinatal risk factors associated with its occurrence.

\section{METHODS}

A retrospective chart review was performed of all ELBW infants $(\mathrm{BW}<1000$ grams) admitted to the neonatal intensive care unit at the University of California, Irvine Children's Hospital during a 42-month interval from January 1, 2002 through June 30, 2005. Approval was obtained from University of California Irvine institutional review board (20054440). SIP was diagnosed if the infant had never been fed or received only trophic feeds, had sudden onset of pnuemoperitoneum or a radiographic gasless abdomen, abdominal distention and/or bluish discoloration, and did not exhibit any radiographic evidence of NEC such as pnuematosis in- 
testinalis or portal venous air. Infants with a diagnosis of NEC were excluded. Infants with a perforation secondary to other etiologies such as intestinal atresia or a distal obstruction were also excluded. Infants with a diagnosis of SIP were compared to infants with no perforation (NP).

Demographic, clinical and therapeutic data were collected. These included maternal complications during gestation (polyhydramnios, oligohydramnios, preeclampsia, diabetes, premature rupture of membranes, choreoamnionitis and maternal substance abuse), maternal medications (antenatal steroids, magnesium, terbutaline, indomethacin, antibiotics), postnatal condition (gestational age, birth weight, APGAR scores, need for intubation and resuscitation at birth, umbilical artery and/or vein catheterization) and medications received in first two weeks of life (controls) and/or two weeks prior to SIP. Clinical diagnosis, type and duration of ventilation and history of feeding/feeding intolerance were also recorded.

Statistical comparisons performed were chi square and unpaired t-tests using SPSS version 14, Chicago, Illinois. Statistical significance was set at $p<0.05$. Data are expressed as mean \pm standard error of the mean.

\section{RESULTS}

A total of 204 ELBW infants were identified during the 42-month period. Of these, 26 were determined to have NEC by radiographic or histologic examination and were excluded. Of the remaining 178 infants, thirteen were diagnosed as having SIP at a mean age of onset of $7.9 \pm 1.9$ days. Maternal and patient demographic data are shown in Table $\mathbf{1 .}$ Mean gestational age and birth weight were similar in the two groups. There was a greater percentage of males in the SIP group $(84.6 \%$ vs $49.1 \%, \mathrm{p}=0.025)$.

Table 1. Maternal and Infant Demographics

\begin{tabular}{|l|c|c|c|}
\hline & SIP & NP & $\boldsymbol{p}$ \\
\hline \hline Maternal & & & \\
Maternal age (years) & $26.8 \pm 2.6$ & $27.8 \pm 0.5$ & NS \\
Cesarean birth (\%) & 67.3 & 69.2 & NS \\
\hline Neonatal & & & NS \\
Mean gestational age (weeks) & $25.9 \pm 0.6$ & $26.2 \pm 0.2$ & NS \\
Birth weight (g) & $732 \pm 40$ & $758 \pm 12$ & $<0.025$ \\
Male infant (\%) & 84.6 & 49.1 & NS \\
APGAR <5 at 1 min (\%) & 46.2 & 57.6 & NS \\
APGAR <5 at 5 min (\%) & 7.7 & 18.2 & $<0.05$ \\
Out born (\%) & 61.5 & 39.9 & NS \\
Mortality (\%) & 7.7 & 15.8 & \\
\hline NS=not significant. & \multicolumn{2}{|l}{}
\end{tabular}

Maternal factors are presented in Table 2 and were comparable in the two groups. Clinical diagnosis and course of the two groups are presented in Table 3. There was a higher percentage of PDA $(92.3 \%$ vs $68.5 \%)$ in the SIP group, but this did not reach statistical significance. PDA was diagnosed by echocardiogram and symptomatic PDA was treated with $1-2$ courses of indomethacin $(0.2 \mathrm{mg} / \mathrm{kg}$ every 12 hours for 3 doses). Umbilical arterial and venous catheter placements were similar in the two groups. Eleven out of the thirteen cases with SIP had blood or peritoneal fluid cultures positive for Staphylococcus epidermidis at presentation and two were positive for Candida.

Table 2. Maternal Risk Factors (\%)

\begin{tabular}{|l|c|c|c|}
\hline & SIP & NP & $\boldsymbol{p}$ \\
\hline \hline Polyhydramnios (\%) & 0 & 0.6 & NS \\
\hline Oligohydramnios (\%) & 0 & 7.3 & NS \\
\hline Preeclampsia (\%) & 0 & 17.0 & NS \\
\hline Diabetes (\%) & 0 & 6.1 & NS \\
\hline Premature rupture of membranes (\%) & 23.1 & 21.2 & NS \\
\hline Group B Streptococcus Chorioamnionitis (\%) & 0 & 2.4 & NS \\
\hline Substance abuse (\%) & & & \\
\hline Tobacco & 0 & 2.4 & NS \\
Alcohol & 0 & 0 & NS \\
Amphetamine & 7.7 & 1.8 & NS \\
Cocaine & 7.7 & 3.6 & NS \\
Heroin/Methadone & 0 & 1.2 & NS \\
Marijuana & 7.7 & 1.2 & NS \\
Barbiturates & 0 & 0.6 & NS \\
\hline NS=not significant.
\end{tabular}

Table 3. Neonatal Clinical Course

\begin{tabular}{|l|c|c|c|}
\hline & SIP & NP & $\boldsymbol{p}$ \\
\hline \hline Respiratory distress syndrome (\%) & 73.3 & 76.9 & NS \\
\hline Patent ductus arteriosus (PDA) (\%) & 92.3 & 68.5 & NS \\
\hline PDA Ligation (\%) & 30.8 & 29.1 & NS \\
\hline PDA ligation after indomethacin failure (\%) & 15.4 & 20.6 & NS \\
\hline PDA ligation with no indomethacin (\%) & 15.4 & 6.7 & NS \\
\hline Intraventricular Hemorrhage (\%) & 24.2 & 30.8 & NS \\
\hline Umbilical artery catheter placement (\%) & 53.9 & 62.2 & NS \\
\hline Umbilical vein catheter placement (\%) & 61.5 & 66.7 & NS \\
\hline NS=Not significant. & & &
\end{tabular}

Perinatal exposure to medications is displayed in Table 4. There was a significantly higher use of tertbutaline in mothers of SIP infants (SIP 30.8\% vs NP 9.1\%, $\mathrm{p}=0.025$ ). Early use of indomethacin (0-3 days) was significantly higher in the SIP group (SIP 69.2\% vs 27.9\%, p=0.002). Hydrocortisone was used for treatment of persisting hypotension in the study population at doses of $1 \mathrm{mg} / \mathrm{kg} / \mathrm{dose}$ every $6 \mathrm{hrs}$ for maximum of 4 doses. Use of hydrocortisone was similar in the two groups, but combination of early treatment with hydrocortisone and indomethacin (0-3 days) was higher in the SIP group ( $7.7 \%$ vs $0.6 \%, \mathrm{p}=0.02)$. Betamethasone was used as an antenatal steroid at our hospital and complete and incomplete courses were included for analysis. Combined exposure to antenatal steroids and postnatal indomethacin was higher in the SIP group (0-3 days: $53.0 \%$ vs $15.2 \%, \mathrm{p}=0.001$; 
Table 4. Medications (\%)

\begin{tabular}{|c|c|c|c|c|}
\hline \multicolumn{4}{|l|}{ Maternal Medications (\%) } & \\
\hline $\mathrm{MgSO} 4$ & 38.5 & 40 & NS & $0.94(0.29-2.99)$ \\
\hline Terbutaline & 30.8 & 9.1 & 0.015 & $4.44(1.22-16.18)$ \\
\hline \multicolumn{5}{|l|}{ Neonatal Medications (\%) } \\
\hline Indomethacin (day 0-3) & 69.2 & 27.9 & 0.002 & $5.65(1.66-19.24)$ \\
\hline Indomethacin (day 4-10) & 7.7 & 17.6 & NS & $0.39(0.05-3.13)$ \\
\hline Indomethacin $(0-14)$ & 76.9 & 50.9 & NS & $1.86(0.49-7.01)$ \\
\hline Dobutamine (day 0-14) & 23.1 & 10.9 & NS & $2.31(0.58-9.13)$ \\
\hline Dopamine (day 0-14) & 69.2 & 34.6 & 0.017 & $4.04(1.19-13.69)$ \\
\hline Caffeine (day 0-14) & 69.2 & 69.7 & NS & $1.45(0.38-5.49)$ \\
\hline \multicolumn{5}{|l|}{ Combination Medications (\%) } \\
\hline Maternal steroids + Neonatal indomethacin (0-14 day) & 69.2 & 36.7 & 0.019 & $3.938(1.163-13.334)$ \\
\hline Maternal steroids + Neonatal indomethacin $(0-3$ days $)$ & 61.5 & 15.2 & 0.001 & $8.554(2.594-28.209)$ \\
\hline Maternal terbutaline + antenatal steroid & 30.8 & 6.7 & 0.003 & $6.222(1.650-23.460)$ \\
\hline Neonatal Hydrocortisone (0-14 days) + Neonatal Indomethacin ( $0-14$ days $)$ & 15.4 & 9.1 & NS & $1.1818(0.368-8.982)$ \\
\hline
\end{tabular}

0-14 days: $69.2 \%$ vs $36.4 \%, \mathrm{p}=0.025)$. Hypotension requiring dopamine was significantly higher in the SIP group (SIP 69.2 vs NP 34.6, $\mathrm{p}=0.017$ ).

\section{DISCUSSION}

In our single institution experience over a period of 42 months, we found that SIP in ELBW infants was associated with early treatment with indomethacin for symptomatic PDA. Furthermore, SIP was associated with hypotension in these infants requiring use of dopamine. We also found an association between SIP and tertbutaline used prenatally as a tocolytic. This association has not been previously described. Most of these SIP cases were associated with Staphylococcus epidermidis infections, consistent with previous reports, including one from our institution [4-6]. While there have been several previous studies reporting the association of various risk factors with SIP, the strength of this study is that we comprehensively examined the various prenatal as well as postnatal factors which may be associated with development of SIP in ELBW infants in a single institution.

A bimodal distribution in SIP presentation has been described by Attridge [15] with $1 / 5^{\text {th }}$ of the cohort presenting in days 0-3 and the remainder between 4-14 days. Interestingly, the early SIP cases included larger infants with mean gestational age of 31 weeks (birth weight $1401 \mathrm{~g}$ ) versus 25 weeks (birth weight $775 \mathrm{~g}$ ) for the late SIP cases. The latter group was also more likely to have received indomethacin and steroids. Infants in our study who developed SIP had a mean age of 7.9 days and thus fall in the late SIP presentation category and share similar characteristics with the late SIP infants described by Attridge, including higher likelihood of early exposure to indomethacin and hydrocortisone. One of the reasons we did not have early SIP presenters is that we only included the smaller preterm infants weighing $<1000 \mathrm{~g}$.

Indomethacin, a prostaglandin synthesis inhibitor, is used extensively in the ELBW population for treatment of PDA and has also been used in several centers for intraventricular hemorrhage (IVH) prophylaxis. It has been known to decrease mesenteric blood flow, cause direct mucosal injury as well as increase intestinal contractility, all of which contribute to intestinal damage [9]. Indomethacin has been linked with SIP in several studies [3, 16-18]. In a multi center randomized study [11], indomethacin was administered to all study infants irrespective of presence of symptomatic PDA. As a secondary outcome, there was no difference in occurrence of SIP in indomethacin prophylaxis and control groups. This suggests that indomethacin may be acting synergistically with other risk factors in development of SIP in these infants. Consistent with this concept, we did not find a significant relationship between indomethacin treatment in the first two weeks of life and development of SIP. However, timing of indomethacin in the first three days of life made the relationship highly significant. At our institution, indo- 
methacin is only used for treatment of symptomatic PDA, which may itself be a co-risk factor for development of SIP as suggested previously by Attridge et al. [3]. This would explain the difference in SIP outcomes between the previous study [11] (where indomethacin was administered prophylactically to all infants $<1000 \mathrm{~g}$ ) and this study (where indomethacin was only used for treatment of symptomatic PDA).

Previous studies have linked combined use of indomethacin and hydrocortisone $[3,9]$ or dexamethasone [10, 19] with development of SIP. We found this to be true for combined exposures to indomethacin and hydrocortisone occurring in the first three days of life. Recent studies have cautioned against using indomethacin in infants with elevated endogenous cortisol levels [3,9]. We found significantly greater occurrence of SIP in outborn versus inborn infants. While transport of infants would be expected to be associated with a certain amount of stress, we do not have information regarding their cortisol levels. Hence combined effects of elevated endogenous cortisol levels and indomethacin need to be further studied.

We found an association between intrauterine exposure to tertbutaline (used as a tocolytic for preterm labor) and SIP. Tertbutaline is a beta 2-adrenergic agonist and has not been previously associated with infant morbidity [20]. However, in the fetus, maternal tertbutaline has been associated with tachycardia, hypotension, ileus and hydrops [21]. One or a combination of these may place the fetus at risk for developing SIP after birth. A recent study showed suppression of glomerular function along with decreased systemic blood pressure in rats over expressing beta 2 adrenergic receptors [22]. Intra renal beta 2 adrenergic receptor expression increases with age in children and is up regulated by steroids [22]. In this study, all infants in the SIP group who were exposed to intrauterine tertbutaline were also exposed to antenatal steroids and the combined effect of tertbutaline and antenatal steroids was significant versus controls. This association between tertbutaline ( \pm antenatal steroids) and SIP needs to be further explored and precautions may need to be taken in ELBW infants exposed to intrauterine tertbutaline and antenatal steroids.

Consistent with previous reports [3] we found that hypotension in ELBW infants requiring treatment was associated with SIP. While there is some capacity for mesenteric blood flow autoregulation, in the presence of profound hypotension, net vasoconstriction and decrease in intestinal blood flow are seen [23]. Interestingly, association with SIP was only found with use of dopamine and not with dobutamine. Whether this is a direct effect of dopamine or reflects a state of hemodynamic compromise is difficult to tell. The protocol followed by clinicians at our unit includes use of fluid bolus followed by dopamine. Dobutamine is added for infants with persisting hypotension. Recent studies suggest that dopamine may have deleterious effects on the intestinal mucosal cells related to redistributing blood flow away from the intestinal mucosa or by decreasing directly the cell redox state [24].

While birth asphyxia was reported in a quarter of their group of SIP in the study by Holland et al. [5] we did not find any association between low APGAR scores and SIP. We also did not find an association between umbilical arterial or venous line placements, or modes of ventilation. We did find a gender difference in cases of SIP which was significant for male infants. This has previously been reported $[5,6]$ and may be related genetically to the better survival figures of female versus male infants. While there is concern that caffeine administration may be associated with reduction in mesenteric blood flow velocity $[25,26]$ we did not find any association between use of caffeine in these infants and SIP.

A weakness in our study is its retrospective design. However the incidence of SIP is only $1-7 \%$ and it would be difficult to study SIP prospectively. We looked at 42 months using our electronic medical records data base and found only 13 cases of SIP which were confirmed by a thorough review of the records by a pediatric surgeon and differentiated from NEC.

In conclusion, while indomethacin use in general may not be related to development of SIP, the timing of indomethacin administration appears to be highly significant. Early administration during the first three days of life increases the risk of SIP. In addition, indomethacin appears to act synergistically with other risk factors, such as the presence of symptomatic PDA and co-exposure to antenatal or postnatal steroids, to increase the risk for SIP in ELBW infants. Hypotension requiring use of dopamine in the first two weeks of life is independently related to development of SIP. In addition, prenatal exposure to maternal tertbutaline can increase the risk for SIP.

\section{ACKNOWLEDGEMENT}

Kristy Davis was supported by a research grant from the Dean's Office, University of California, Irvine School of Medicine.

\section{REFERENCES}

[1] Pumberger W, Mayr M, Kohlhauser C, et al. Spontaneous localized intestinal perforation in very-low-birth-weight infants: a distinct clinical entity different from necrotizing enterocolitis. J Am Coll Surg 2002; 195: 796-803.

[2] Buchheit JQ, Stewart DL. Clinical comparison of localized intestinal perforation and necrotizing enterocolitis in neonates. Pediatrics 1994; 93: 32-6.

[3] Attridge JT, Clark R, Walker MW, et al. New insights into spontaneous intestinal perforation using a national data set: (1) SIP is associated with early indomethacin exposure. J Perinatol 2006; 26 : 93-9.

[4] Novack CM, Waffarn F, Sills JH, et al. Focal intestinal perforation in the extremely-low-birth-weight infant. J Perinatol 1994; 15: 45053.

[5] Holland AJA, Shun A, Martin HCO, et al. Small bowel perforation in the premature neonate: congenital or acquired? Pediatr Surg Int 2003; 19: 489-94.

[6] Mintz AC, Applebaum H. Focal gastrointestinal perforations not associated with necrotizing enterocolitis in very low birth weight neonates. J Pediatr Surg 1993; 28: 857-60.

[7] Parrish RA, Sherman RT, Wilson H. Spontaneous rupture of the gastro-enteric tract in the newborn: a report of 13 cases and description of a characteristic X-ray finding. Ann Surg 1964; 159: 244-51.

[8] Salter AEH. Congenital rupture of jejunum. Med J Aust 1930; 1: 422-23.

[9] Watterberg KL, Gerdes JS, Cole CH, et al. Prophylaxis of early adrenal insufficiency to prevent bronchopulmonary dysplasia: a multicenter trial. Pediatrics 2004; 114: 1649-57.

[10] Stark AR, Carlo WA, Tyson JE, et al. Adverse effects of early dexamethasone treatment in extremely low birth weight infants. N Engl J Med 2001; 344: 95-101. 
[11] Schmidt B, Davis P, Moddemann D, et al. Long-term effects of indomethacin prophylaxis in extremely-low-birth-weight infants. N Eng1 J Med 2001; 344: 1966-72.

[12] Bloom BT. Letter. J Perinatol 2006; 26: 384.

[13] Kawase $\mathrm{Y}$, Ishii $\mathrm{T}$, Arai $\mathrm{H}$, et al. Gastrointestinal perforation in very low-birth weight infants. Pediatr Int 2006; 48: 599-603.

[14] Attridge JT, Clark R, Walker MW, et al. New insights into spontaneous intestinal perforation using a national data set: (3) antenatal steroid have no adverse association with spontaneous intestinal perforation. J Perinatol 2006; 26: 667-70.

[15] Attridge JT, Clark R, Walker MW, Gordon PV. New insights into spontaneous intestinal perforation using a national data set: (2) two populations of patients with perforations. J Perinatol 2006; 26: 18588.

[16] Alpan G, Eyal F, Vinograd I. Localized intestinal perforations after enteral administration of indomethacin in premature infants. J Pediatr 1985; 106: 277-81.

[17] Nagaraj HS, Sandhu AS, Cook LN, et al. Gastrointestinal perforation following indomethacin prophylaxis in extremely-low-birthweight infants. J Pediatr Surg 1981; 16: 1003-1007.

[18] Scholz TD, McGuinness GA. Localized intestinal perforation following intravenous indomethacin for patent ductus arteriosus. J Pediatric Gastroenterol Nutr 1988; 7: 773-5.
[19] Paquette L, Friedlich P, Ramanathan R, Seri I. Concurrent use of indomethacin and dexamethasone increases the risk of spontaneous intestinal perforation in very low birth weight neonates. J Perinatol 2006; 26: 486-92.

[20] Anotayanonth S, Subhedar NV, Garner P, et al. Betamimetics for inhibiting preterm labor. Cochrane Database Syst Rev 2004; 4: CD004352.

[21] Norwitz ER, Robinson JN, Challis JRG. The control of labor. N Engl J Med 1999; 341: 660-66.

[22] Nakamura A, Niimi R, Imaizumi A, et al. Renal effects of beta2adrenoceptor agonist and the clinical analysis in children. Pediatr Res 2007; 61: 129-33.

[23] Clark DA, Munshi UK. Fetal and neonatal physiology. In: Polin RA, Fox WW, Abman SH (Eds). Saunders, Philadelphia, 2004.

[24] Guerin JP, Levraut J, Samat-Long C, et al. Effects of dopamine and norepinephrine on systemic and hepatosplanchnic hemodynamics, oxygen exchange, and energy balance in vasoplegic septic patients. Shock 2005; 23: 18-24.

[25] Hoecker C, Nelle M, Poeshl J, et al. Caffeine impairs cerebral and intestinal blood flow velocity in preterm infants. Pediatrics 2002; 109: 784-87.

[26] Lane AJ, Coombs RC, Evans DH, et al. Effect of caffeine on neonatal splanchnic blood flow. Arch Dis Child Fetal Neonatal 1999; Ed 80: F128-F29. 